\title{
Risk Factors for Acquiring Multi-Drug Resistant Klebsiella Pneumoniae At A Tertiary Teaching Hospital in China
}

\section{Weidong Zhang}

traditional chinese hospital of LuAn

\section{Yuan Gao}

Maternity\&Child care center of DeZhou

Shuo Huang

hunan provincial maternal and child health care hospital

Anhua Wu

Xiangya Hospital Central South University

chenchao fu ( $\nabla$ fuchenchao112@163.com )

Xiangya Hospital Central South University https://orcid.org/0000-0003-4137-7587

\section{Research}

Keywords: Klebsiella Pneumoniae, Drug Resistant, Transformation, Risk Factors

Posted Date: March 26th, 2021

DOl: https://doi.org/10.21203/rs.3.rs-322693/v1

License: (c) (i) This work is licensed under a Creative Commons Attribution 4.0 International License. Read Full License 


\section{Risk factors for acquiring multi-drug resistant klebsiella pneumoniae at a tertiary teaching hospital in China}

Zhang Weidong1, Gao Yuan2, Huang Shuo3,Wu Anhua4, Fu Chenchao4*

Zhang Weidong and Gao Yuan have equal contribution to this article.

1 Traditional Chinese Hospital of Luan, Luan 237000, China

2 Maternity\&Child Care Center of Dezhou, Dezhou 253015, China

3 Hunan Provincial Maternal and Child Health Care Hospital, Changsha 410008, China

4 Xiangya Hospital, Central South University, Changsha 410008, China

Corresponding author: Fu chenchao, Xiangya Hospital, Central South University, Changsha 410008, China. Email: fuchenchao112@163.com

\section{Abstract}

BACKGROUD To analyze the risk factors for acquiring multi-drug resistant Klebsiella pneumoniae (MDR-Kpn).

METHODS Data were collected from patients who were admitted into a tertiary teaching hospital in China from January 2018 to October 2020. Sensitive Klebsiella pneumoniae (Kpn) was detected in 82 patients. After a period of treatment, 41 patients were detected with MDR-Kpn, and they were designed as the MDR-Kpn group. Another 41 patients always detected with sensitive Klebsiella pneumoniae were assigned to the sensitive Kpn group. The baseline characteristics and clinical parameters were compared between these two groups. Multivariate logistic regression analysis were conducted to determine the risk factors for acquiring MDR-Kpn. RESULTS The proportion of sex, history of smoking and department distribution had no significant differences between these two groups. However, the patients with chronic pulmonary diseases and neurological disorders were more likely to acquire MDR-Kpn. It also showed longer hospital stay before the first detection of multi-drug resistant Kpn in MDR-Kpn group than the hospital stay before the last detection of sensitive-Kpn in sensitive Kpn group. Additionally, days of hospitalization, ICU stay, days of drainage tube use, times of sputum suction, days of foley catheter use, days of vascular catheter use between the twice detection (it represents the time interval between first detection of sensitive-Kpn to first detection of resistant-Kpn in MDR-Kpn group, and also represents the time interval between first detection of sensitive-Kpn to last detection of sensitive-Kpn in sensitive Kpn group), were higher in MDR-Kpn group. The results showed more categories of antimicrobials in patients of acquiring MDR-Kpn, along with more days of using broad-spectrum cephalosporins, fluoroquinolones and glycylcyclines, compared to non-transition group. Multivariate logistic regression analysis showed that the number of comorbidities, hospital stay before the first detection of multidrug-resistan Kpn (MDR-Kpn group) or last detection of sensitive Kpn (Sensitive group), days of using 
drainage tube and number of antimicrobial categories between the two detection were independent risk factors of acquiring multidrug-resistance for Kpn.

CONCLUSION: The number of comorbidities, the hospital stay, the number of antimicrobial categories, and days of using drainage tube were independent risk factors for acquiring MDR-Kpn.

Key words: Klebsiella pneumoniae; Drug resistance; Transformation; Risk factors

\section{Introduction}

Klebsiella pneumonia (Kpn) is a common pathogen that causes a wide range of nosocomial and community-acquired infections, and it accounts for more than $95 \%$ of Klebsiella infections. When the host's immune system is compromised or skin tissue is damaged, the pathogen can invade the host and cause infections through respiratory tract, blood, urinary system, etc, resulting in prolonged hospital stay and increased medical expense. What's more, multi-drug resistant (MDR) strains of Klebsiella pneumoniae has been increasingly detected in recent years, and it has become one of the most important pathogens causing nosocomial infections (hospital-acquired infection) and seriously threatened the lives of patients [1-2]. Clinically, carbapenem antibiotics, with the advantages of broad antibacterial spectrum and strong antibacterial activity, have become the main therapeutic drugs for Klebsiella pneumoniae infection. However, along with more and more normalized application of carbapenem antibiotics, the status of MDR-Kpn is getting worse, thus resulting in more failure of clinical treatment and death [3]. Results from China Antimicrobial Surveillance Network (CHINET) showed that the rate of carbapenems-resistant Kpn rose from $3.6 \%$ in 2009 to $24.6 \%$ in 2018 [4]. Patients infected with carbapenem-resistant Kpn usually progressed and deteriorated rapidly, with a high mortality rate between $40 \%$ to $70 \%$. Additionally, majority of carbapenem-resistant Kpn were detected in critically ill patients from intensive care unit (ICU). The factors including greater severity of disease and poor immune system in critically ill patients, made them have a propensity to acquire carbapenem-resistant Kpn than those patients from other departments, and carbapenem-resistant Kpn has become a critical risk factor for hospital mortality [5]. Most studies focused on the relationship between the acquisition of drug-resistant Kpn and the use of invasive procedures and antimicrobial agents during whole hospitalization. In the current study, we set up a time point segmentation of Klebsiella pneumoniae transforming from susceptibility to multiple commonly used antimicrobial agents to multi-drug resistant Kpn (MDR-Kpn), and focused on association between MDR-Kpn acquisition and various invasive operation and treatment strategies during a specific period, and explored the susceptible risk factors of acquiring multi-drug resistance for $\mathrm{Kpn}$, and provided a more accurate reference for MDR-Kpn prevention and control. 


\section{Methods}

\subsection{Study design and participants}

This was a retrospective cohort study of hospitalized patients admitted to Xiangya Hospital, Central South University from January 1, 2018 to October 31, 2020. The patients were firstly detected with sensitive Klebsiella pneumoniae. After a period of treatment, 41 patients were detected with multi-drug resistant Klebsiella pneumoniae, and these patients were divided into MDR-Kpn group. Another 41 patients were still detected with sensitive Klebsiella pneumoniae during the same period, and they were assigned to sensitive Kpn group. This study's protocol was reviewed and approved by the Ethics Commission of Xiangya Hospital of Central South University, and the written informed consent was obtained from each patients.

\subsection{Data collection}

Demographic (age and sex) and clinical data (smoking, comorbidities, length of hospital stay, hospital stay from admission to first detection of MDR-Kpn or hospital stay from admission to last detection of sensitive Kpn, period between the first detection of sensitive Klebsiella pneumoniae and first detection of MDR-Kpn, and period between the first detection of sensitive Klebsiella pneumoniae and last detection of sensitive Kpn in the two groups respectively) were collected from the electronic medical record system. The length of ICU stay, use of invasive mechanical ventilation and tracheotomy, days of using ventilator and drainage tubes (including abdominal drainage tube, ventricular drainage tube, thoracic drainage tube, anal canal and wound drainage), days of using a gastric tube, times of sputum suction, and days of using a foley catheter or a vascular catheter, were also collected. Additionally, the data related to the use of antibiotics including the type and length of antimicrobial agents used between the two samples of detection were obtained as well.

\subsection{Measurements}

The specimens (including sputum, blood, urine, etc) were collected, and the bacteria were isolated, cultured and identified in accordance with the fourth edition of The National Clinical Laboratory Operating Procedures. The bacterial strain identification and drug susceptibility test were carried out using the automatic microbial identification and analysis system (Vitek 2 Compact). The quality control strain KLEbsiella pneumoniae ATCC700603 was purchased from the Clinical Testing/Laboratory Center of the Ministry of Health and the drug susceptibility was interpreted according to the 2019 American Association for Clinical Laboratory Standardization (CLSIM100-S29). 


\subsection{Criteria for MDR-Kpn}

If the isolated Kpn is not sensitive to three or more antimicrobial classes in eleven classes of antimicrobial agents (including non-broad-spectrum cephalosporins, broad-spectrum cephalosporins, penicillins, penicillins plus enzyme inhibitors, carbapenems, monocyclic $\beta$-lactams, fluoroquinolones, tetracyclines, amino Glycosides, folate metabolism inhibitors, glycylcyclines) for Kpn, it is defined as MDR-Kpn [6]. Since Kpn is naturally resistant to pseudomonas penicillin and enzyme inhibitors, these classes of antimicrobials are not included.

\subsection{Statistical analysis}

Continuous variables following a normal distribution were expressed as the mean and standard deviation and analyzed using a Student's t-test. In contrast, those with a skewed distribution were presented as medians and interquartile ranges (IQR) and analyzed using the Mann-Whitney U test. Categorical variables were summarized as counts and proportions and evaluated using the Chi-square test. Multiple logistic regression analysis was conducted to determine the risk factors for acquiring multi-drug resistance of klebsiella pneumoniae. All statistical analyses and graphs were generated using SPSS version 25.0 (IBM, United States), and a P-value $<0.05$ was accepted to be statistically significant.

\section{Results}

3.1 General information of the included patients

The characteristics of the included patients were summarized in Table 1. Sixty-four males and 18 females were included and analyzed in the current study. The median age was 53 years (range from 0 to 92 years) in MDR-Kpn group, and 55 years (rang from 0 to 96 years) in sensitive group. The proportion of sex, history of smoking and department distribution had no significant differences between these two groups. However, the patients with comorbidities of chronic pulmonary diseases $(\mathrm{P}=0.02)$ and neurological disorders $(\mathrm{P}=0.00)$ were more likely to acquire multidrug-resistant Klebsiella pneumoniae.

\subsection{Clinical parameters and treatment strategies of the included patients}

The clinical parameters were compared between these two groups in Table 2, and the results showed that patients in MDR-Kpn group had more underlying chronic comorbidities $(\mathrm{P}=0.009)$ and treatments of blood transfusion $(\mathrm{P}=0.01)$. It also showed longer hospital stay before the detection of resistant-Kpn in patients of MDR-Kpn group than the hospital stay before the last detection of sensitive-Kpn in patients of sensitive group ( $\mathrm{P}=0.007)$. Additionally, days of hospitalization ( $\mathrm{P}=0.000)$, ICU stay 
between the twice detection (it represents the time interval between first detection of sensitive-Kpn to first detection of resistant-Kpn in MDR-Kpn group, and also represents the time interval between first detection of sensitive-Kpn to last detection of sensitive-Kpn in sensitive group) ( $\mathrm{P}=0.047)$, days of the drainage tube use $(\mathrm{P}=0.013)$, times of sputum suction $(\mathrm{P}=0.033)$, days of foley catheter use $(\mathrm{P}=0.041)$ and days of vascular catheter use $(\mathrm{P}=0.043)$ between the twice detection, were also different between these two groups.

\subsection{Analysis of the use of antimicrobial agents.}

The analysis of the use of antibiotics during the twice detection between these two groups was shown in Table 3. The results showed more types of antibiotics were used in patients of MDR-Kpn group compared to sensitive group $(\mathrm{P}=0.00)$. Additionally, patients in MDR-Kpn had more days of using broad-spectrum cephalosporins $(\mathrm{P}=0.01)$, fluoroquinolones $(\mathrm{P}=0.03)$ and glycylcyclines $(\mathrm{P}=0.04)$.

\subsection{Multivariate logistic regression analysis on risk factors of acquiring MDR-Kpn} Multivariate logistic regression was summarized in Table 4 after including the above susceptible factors that were significantly different between these two groups. The results showed that the number of comorbidities (OR 1.629, 95\%CI 1.081-2.456), hospital stay before the first detection of multidrug-resistan Kpn (MDR-Kpn group) or last detection of sensitive Kpn (Sensitive group) (OR 1.045, 95\%CI 0.998-1.095), days of using drainage tube (OR 1.106, 95\%CI 1.012-1.209) and number of antimicrobial categories between the two detection (OR 2.228, 95\% CI 1.279-4.094) (it represents the time interval between first detection of sensitive-Kpn to first detection of resistant-Kpn in MDR-Kpn group, and also represents the time interval between first detection of sensitive-Kpn to last detection of sensitive-Kpn in sensitive Kpn group) were independent risk factors of acquiring multidrug-resistance for Kpn.

\section{Discussion}

Klebsiella pneumoniae belongs to the most common strain of bacteria in the genus Klebsiella bacillus, ranking the second in bloodstream infections and the first in respiratory infection with hospital-acquired gram-negative bacteria [7-8]. Along with the extensive and unreasonable use of antibiotics in recent years, multidrug-resistant Klebsiella pneumoniae has be increasingly isolated, resulting in more difficulties of controlling clinical infections and problems of public health around the world [9]. Previous studies reported that higher prevalence of MDR-Kpn was seen in ICU patients, and those critically ill patients with long-term hospitalizations and older ages had a propensity to acquire MDR-Kpn because of the decreased sensitivity to 
antibiotics and weakened immune systems [10-11]. Carbapenems are the first choice for the treatment of Klebsiella pneumoniae, and their broad antibacterial spectrum and strong antibacterial activity make them become one of the important antibiotics for the treatment of severe bacterial infections. However, with the extensive use of multiple antimicrobials, the problem of carbapenem-resistant Kpn has emerged become the biggest challenge for the treatment of Klebsiella pneumoniae around the world [12].

The current study showed that the number of chronic comorbidities, hospital stay before first detection of multidrug-resistant Kpn (MDR-Kpn group) or last detection of sensitive Kpn (Sensitive Kpn group), days of using drainage tube and the number of antimicrobial categories between the two detection were independent risk factors of acquiring multi-drug resistance for Kpn. Patients with MDR-Kpn had higher prevalence of chronic pulmonary disease, which could explain that Kpn was the main pathogen in patients with hospital or community-acquired gram-negative bacillus pneumonia. A higher prevalence of neurological disorders was also observed in patients with MDR-Kpn, since those patients with neurological disorders of consciousness and mobility were more likely to be affected by MDR-Kpn. As many patients have several pre-existing comorbidities, the multivariate logistic regression analysis showed that the number of comorbidities was an independent risk factor of acquiring multi-drug resistance for Kpn. Therefore, it could be deliberately concluded that the patients with more comorbidities, greater severity and worse immunity, were more likely to acquire the multi-drug resistance from sensitive Kpn.

Previous studies showed that the length of hospital stay was an important risk factor of carbapenem-resistant Kpn infection [13]. Prolonged hospitalization, accompanied with various operations and treatments, would make patients be more susceptible to MDR-Kpn. However, previous retrospective investigations mainly focused on entire course of hospital stay, and did not divided the phases of hospitalization based on the timepoint of acquiring MDR-Kpn. In the current study, the patients in MDR-Kpn group had significantly longer hospital stay than those patients in sensitive Kpn group. Additionally, the hospital stay before the first detection of resistant-Kpn in MDR-Kpn group was longer than the hospital stay before last detection of sensitive Kpn in the control group, and the multivariate logistic regression analysis demonstrated it was an independent risk factor of acquiring multi-drug resistance for $\mathrm{Kpn}$. Therefore, it is important and urgent to identify the patients with high risk of MDR-Kpn to reduce the incidence of acquiring multi-drug resistance for Kpn and shorten hospital stay with early and effective treatments [14].

Some studies have shown that the use of invasive medical procedure was an independent risk factor of Kpn colonization or infection [15]. The use of urinary catheter was an independent risk factor of multi-drug resistant bacteria infection in 
ICU patients [16]. In addition, surgery damaged the skin barrier and provided more chances for multi-drug resistant bacteria to invade into the bodies [16]. Long-term tracheal intubation was more likely to form a biofilm on the inner wall of the artificial airway, and increase colonization and infection of multi-drug resistant strains [17]. Long-term use of drainage tube always causes congestion and edema in surrounding tissues, and makes it easier for bacteria to colonize and increases the risk of retrograde infection and bloodstream infection [18]. Frequent maintenance also increases the chance of invasive infections. In this study, we focused on the time and frequency of using various invasive operations (including ventilators, urinary catheters, vascular catheters, sputum suction, gastric tube and drainage tube). Although most above variates had a significant difference between the patients with or without acquiring MDR-Kpn, the multivariate regression analysis showed only the days of using drainage tube was an independent risk factor for acquiring MDR-Kpn after adjusting these confounding factors.

At present, the types of antimicrobials used in the treatment of Klebsiella pneumoniae mainly include $\beta$-lactams, quinolones, aminoglycosides, glycylcyclines, and polymyxins. Previous studies showed that the use of antibiotics, such as cephalosporins, carbapenems and quinolones, was an independent risk factor for acquiring MDR-Kpn [19-21]. In the present study, the data showed more days of using broad-spectrum cephalosporins, fluoroquinolones and glycyrrhizin, and greater number of antimicrobial categories in patients with MDR-Kpn. However, the multivariate logistic regression analysis showed that only the number of antimicrobial categories was an independent risk factor for acquiring MDR-Kpn after adjusting those confounding factors. Sensitive Kpn is characterized with strong ability of acquiring mutation and resistance, and it is more likely for patients to acquire MDR-Kpn after a long-term use of various antibiotics. Therefore, using antimicrobials prudently and reasonably would be the best way to reduce the chances of acquiring MDR-Kpn.

\section{Conclusions}

Collectively, in the treatment of sensitive Kpn colonization or infection, physicians should pay more attention to enhance the immunity of patient while treating their primary disease. Various invasive treatments should be carefully and comprehensively evaluated before the operation. It must be subject to the aseptic principles for invasive procedure, and their regular maintenance and replacement. A comprehensive and timely assessment is also required to help remove the drainage tube as soon as possible. Active surveillance to identify MDR-Kpn is a recommended strategy for early control and treatment of MDR-Kpn. When choosing combined antibiotics for sensitive Kpn, physicians should pay more attention to the number of antimicrobial 
categories.

\section{References}

[1] Ferreira C M , Ferreira W A , Silva L M , et al.Molecular Epidemiology of KPC-2 Producing Klebsiella pneumoniae[J].SOJ Microbiology \& Infectious Diseases , 2017 ， $5(1): 1-3$.

[2] Jiménez ,Adriana ,Castro ,José G ,Munoz-Price L S ,et al. Outbreak of Klebsiella pneumoniae Carbapenemase-Producing Citrobacter freundii at a Tertiary Acute Care Facility in Miami , Florida[J].Infection Control \& Hospital Epidemiology , 2017 , 38(03):320-326.【PMID : 27923418】

[3] Kobayashi S D , Porter A R , Freedman B , et al.Antibody-Mediated Killing of Carbapenem-Resistant ST258 Klebsiella pneumoniae by Human Neutrophils[J].Mbio , $2018,9(2): 1615-1622$.

[4] 2017 CHINET China Bacterial Resistance Surveillance[J]. Chinese Journal of Infection and Chemotherapy, 2018, 18(03):241-251.

[5] Kobayashi S D , Porter A R , Freedman B , et al.Antibody-Mediated Killing of Carbapenem-Resistant ST258 Klebsiella pneumoniae by Human Neutrophils [J]. Mbio,2018, 9(2):1615-1622.

[6] Magiorakos A P, Srinivasan A, Carey R al. Multidrug $\neg$ resistant (MDR), extensively drug-resistant (XDR) and pan $\neg$ drug-resistant (PDR) bacteria in healthcare settings. Expert proposal for a standardized international terminology [ EB/ OL]. (2010 - 08). ECDC Entwurf 2010 , www. escmid. org.

[7] Raza S , Mohsin M , Madni W A , et al.First Report of blaCTX-M-15-Type ESBL-Producing Klebsiella pneumoniae in Wild Migratory Birds in Pakistan[J]. Ecohealth , $2017,14(1): 1-5$.

[8] Kim J O , Song S A , Yoon E J , et al.Outbreak of KPC-2-producing Enterobacteriaceae caused by clonal dissemination of Klebsiella pneumoniae ST307 
carrying an IncX3-type plasmid harboring a truncated Tn4401a[J].Diagn Microbiol Infect Dis , $2017,87(4): 343-348$.

[9] Venieri D , Gounaki I , Bikouvaraki M , et al.Solar photocatalysis as disinfection technique: Inactivation of Klebsiella pneumoniae in sewage and investigation of changes in antibiotic resistance profile[J].Journal of Environmental Management, $2017,195(2): 140-147$.

[10] Margaret M C L , Wyres K L , Duchêne Sebastian , et al. Population genomics of hypervirulent Klebsiella pneumoniae clonal group 23 reveals early emergence and rapid global dissemination[J]. Nature Communications , 2018 , 9(1):2703-2711.

[11] M , Souli , I , et al.Double-carbapenem combination as salvage therapy for untreatable infections by KPC-2-producing Klebsiella pneumoniae[J].European Journal of Clinical Microbiology \& Infectious Diseases , $2017,36(7): 1305-1315$. [12] Abderrahim A , Djahmi N , Pujol C , et al.First Case of NDM-1-Producing Klebsiella pneumoniae in Annaba University Hospital, Algeria[J].Microbial Drug Resistance , 2017 , 16(7):78-81.

[13] Banach DB , et al. Active Surveillance for Carbapenem-Resistant Entero bacteriaceae using Stool Specimens Submitted for Testing for Clostridium difficile[M].Infect Control Hosp Epidemiol 2014(35): 82-84.

[14] Mendes Ana Constan?a , Novais ?ngela , Campos Joana, et al.mcr-1 in Carbapenemase-Producing Klebsiella pneumoniae with Hospitalized Patients , Portugal，2016-2017.[J].Emerging Infectious Diseases , $2018 ， 24(4): 762-766$.

[15] Akturk H ,Sutcu M ,Somer A ,et al.Carbapenem-resistant Klebsiella pneumoniae colonization in pediatric and neonatal intensive care units : Risk factors for progression to infection[J].J Infect Dis , 2016,20 ( 2 ) :134-140. 
[16] Aljanaby AA J , Alhasnawi H M R J. Phenotypic and Molecular Characterization of Multidrug Resistant Klebsiella pneumoniae Isolated from Different Clinical Sources in Al-Najaf Province-Iraq[J].Pakistan Journal of Biological Sciences , 2017 , 20(5):217-232.

[17] Tang W ,Cheng JN ,Yu Y ,et al.Effect of bacterial biofilm on multidrug resistance of Escherichia coli[J]. Chinese Journal of Nosocomial Infection,2019， 029(003):326-329.

[18] Shirata C , Hasegawa K , Kokudo T , et al.Surgical Site Infection after Hepatectomy for Hepatocellular Carcinoma[J].Dig Surg , 2018 ，35(3):204-211.

[19] Kwak YG , et al.Risk factors for the acquisition of carbapenem-resistant Klebsiella pneumoniae among hospitalized patients[M].Microb Drug Resist 2005(11): 165-169.

[20] Gasink LB , et al.Risk factors and clinical impact of Klebsiella pneumoniae carbapenemase-producing K.pneumoniae[M].Infect Control Hosp Epidemiol 2009 (30):1180-1185.

[21]Schechner V , et al.Predictors of rectal carriage of carbapenem-resistant Enterobacteriaceae (CRE) among patients with known CRE carriage at their next hospital encounter[M].Infect Control Hosp Epidemiol 2011(32): 497-503. 


\section{Supplementary Files}

This is a list of supplementary files associated with this preprint. Click to download.

- Table1.Baselinecharacteristics.pdf

- Table2.Clinicalparameters.pdf

- Table3.Analysisofantibacteriause.pdf

- Table4.Multivariatelogisticregression.pdf 